\title{
Young open clusters in the Milky Way and Small Magellanic Cloud
}

\author{
C. Martayan \\ ${ }^{1}$ European Organisation for Astronomical Research in the Southern Hemisphere, \\ Alonso de Cordova 3107, Vitacura, Casilla 19001, Santiago 19, Chile \\ email: cmartaya@eso.org \\ ${ }^{2}$ GEPI, Observatoire de Paris, CNRS, Université Paris Diderot, 5 place Jules Janssen, \\ 92195 Meudon Cedex, France
}

\begin{abstract}
NGC 6611, Trumpler 14, Trumpler 15, Trumpler 16, and Collinder 232 are very young open clusters located in star-formation regions in the Eagle Nebula and Carina in the Milky Way, and NGC 346 in the Small Magellanic Cloud. With different instrumentation and techniques, it has been possible to detect and classify new Herbig Ae/Be and classical Be stars and to provide new tests/comparisons of the Be stars' appearance models. Special (He-strong) stars in these star-formation regions are also discussed.
\end{abstract}

Keywords. open clusters and associations: individual (Trumpler 14, Trumpler 15, Trumpler 16, Collinder 232), Magellanic Clouds, galaxies: star clusters, stars: early-type, stars: pre-mainsequence, stars: emission-line, Be, stars: evolution, dust, extinction

\section{Observations}

We used the ESO-WFI (Baade et al. 1999) in slitless mode (focusing on $\mathrm{H} \alpha, R \sim 1000$ ) to observe the Galactic open cluster NGC 6611 in the Eagle Nebula. This setup allows us to disentangle the stars, based on emission lines from their circumstellar disks, from the nebular lines due to diffuse emission in the surrounding nebula. We observed 100 stars in NGC 6611, pre-selected from our WFI catalogue, using the VLT-FLAMES/GIRAFFE facilities in MEDUSA mode (Pasquini et al. 2002). We also pre-selected B-type stars in the open clusters Trumpler 14, 15, 16, and Collinder 232 in the Carina region from our astrophotometric catalogues based on the EIS pre-FLAMES survey images (Momany et al. 2001). We then observed $\sim 200$ OB-type stars with VLT-FLAMES/GIRAFFE. We used the LR2, HR4, HR5, HR6, and HR15 settings with resolutions from $R=6500$ to 18000 . These setups were chosen for the Balmer lines, Helium I and II, Mg II, and Si III. The open cluster NGC 346 in the Small Magellanic Cloud was also observed with the ESO-WFI in slitless mode $(\mathrm{H} \alpha)$. More than 50 emission-line stars were found, half of them were detected for the first time: see Martayan et al. (2009).

\section{Determination of the interstellar reddening and correction of the magnitudes}

To correct the magnitudes of the stars for interstellar reddening, we measured the equivalent widths of the interstellar lines at 443.0, 450.2, and $661.3 \mathrm{~nm}$. We then used the calibration from Herbig (1975) to obtain the values of the interstellar reddening, $E(B-V)$ for each star observed with GIRAFFE. A reddening map for Trumpler 14, 15 , and 16 is shown in Figure 1. Based on this estimate of $E(B-V)$, we corrected the 2mass $J H K$ magnitudes as well as the Spitzer magnitudes. However, Spitzer data 


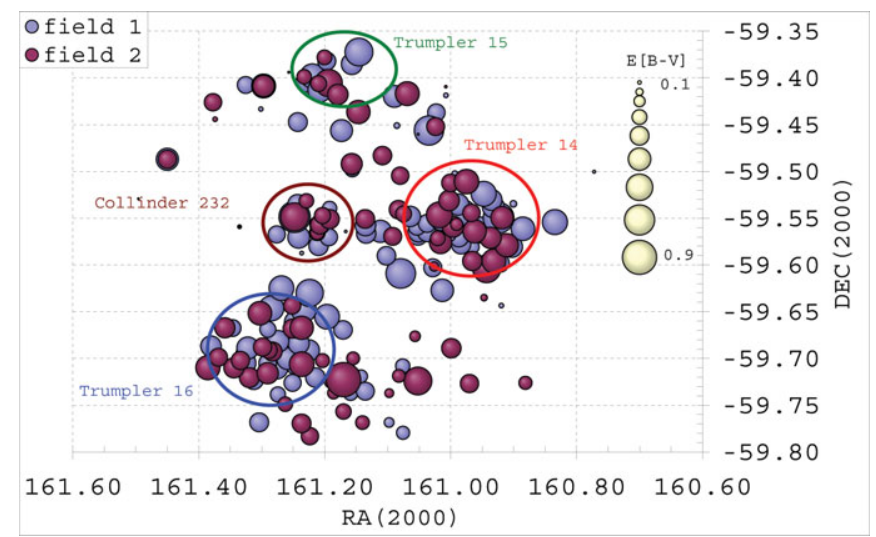

Figure 1. Map of the interstellar reddening in the Carina region for stars observed in Trumpler 14, 15, 16, and Collinder 232.

are only available for the stars in NGC 6611 but not for those in Trumpler 14, 15, and 16. Two kinds of dereddened colour-magnitude diagrams were obtained for NGC 6611 (see Martayan et al. 2008) For the Trumpler 14, 15, and 16 clusters, the 2MASS diagram is shown in Figure 2. The emission-line stars are indicated by red triangles. The status of several of these is obvious: they are clearly pre-main-sequence (pre-MS) or MS objects. However, for the stars located in the intermediate regions, complementary analysis must be done, using the evolutionary status determined from the fundamental stellar parameters and compared to the age of the open clusters.

\section{Determination of fundamental parameters}

The fundamental parameters were determined using the GIRFIT code of Frémat et al. (2006). This code fits the observed spectrum with theoretical templates obtained from the TLUSTY and SYNSPEC codes of Hubeny \& Lanz (1995). For each open cluster member star, we obtained the most appropriate age using theoretical evolutionary tracks

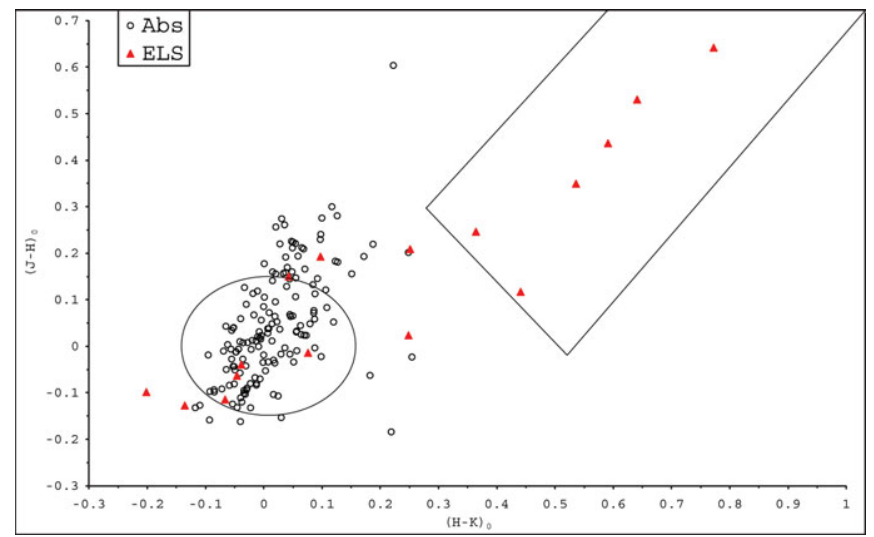

Figure 2. Dereddened 2MASS colour-colour diagram for stars observed in the Trumpler 14, 15, 16, and Colinder 232 open clusters. The large rectangular area from Hernández et al. (2005) corresponds to the area containing pre-main-sequence objects like Herbig Ae/Be stars. 
from Schaller et al. (1992). The open cluster age was then redetermined using these stars.

\section{Nature of the emission-line stars}

To determine the exact nature of the emission-line objects in NGC 6611, Trumpler 14, 15, 16, and Collinder 232, we combined the different parameters from the colour-colour diagrams with the evolutionary status determined based on the Hertzsprung-Russelldiagram evolutionary tracks:

- if the star is located in the 'box' outlining Herbig Ae/Be stars from Hernández et al. (2005) in the dereddened 2MAss diagram, then the star is a pre-MS star.

- if the star is located in the class I or II areas in the Spitzer diagram, then the star is a pre-MS star.

- if the star is located in an intermediate region, the evolutionary status obtained from the fundamental parameters combined with their membership of a given open cluster must be taken into account:

o open cluster member with an age equivalent to that of the cluster: MS star;

o open cluster member with an apparently older age than that of the cluster: pre-MS star;

o not a member of the open cluster: uncertain.

- if the star is not present in the boxes of pre-MS stars and if its evolutionary status is incompatible with the age of its host open cluster, it is probably a classical Be star (with a decretion disk instead of an accretion disk for pre-MS stars like Herbig Ae/Be stars).

All details regarding NGC 6611 are available in Martayan et al. (2008).

\section{Special stars}

Among the emission-line stars of NGC 6611, two are of special interest. The first is W601, which was found to host a magnetic field by Alecian et al. (2008). They also found that this star is a He-strong star. Thanks to VLT-GIRAFFE observations of W601 and W080, it was possible to compare the spectral features of these two stars. We found that W080 is a spectral twin of W601 and is probably also a He-strong star. Moreover, its broad, deep spectral lines cannot be reproduced by the models (Martayan et al. 2008). To discover whether W080 hosts a magnetic field like W601, two sets of observations were obtained at the CFHT with the ESPADONS spectropolarimeter. However, the faintness of W080 implies that this star is at the limit of the capabilities of this instrument. The preliminary results seem to show no strong magnetic field in W080. However, the data are currently being analysed to find a potentially weaker magnetic field and/or to provide upper limits to its intensity as well as confirmation of its He-strong status.

\section{Conclusions}

We found 11 emission-line stars in NGC 6611 and its vicinity, nine of whih are new Herbig Ae/Be stars. In the open clusters Trumpler 14, 15, 16, and Collinder 232 and their vicinity in the Carina nebula, six of the 16 emission-line stars seem to be Herbig Ae/Be stars. For 10 of the remaining emission-line stars, the status is uncertain for two and eight are probably MS and hence classical Be stars. In NGC 346, complementary observations at higher spectral resolution are needed to determine the exact nature of the 50 emission-line stars found. 


\section{References}

Alecian, E., et al. 2008, A\&A (Letters), 481, L99

Baade, D., et al. 1999, ESO Messenger, 95, 15

Frémat, Y., Neiner, C., Hubert, A.-M., Floquet, M., Zorec, J., Janot-Pacheco, E., \& Renan de Medeiros, J. 2006, A\&AA, 451, 1053

Herbig, G. H. 1975, ApJ, 196, 129

Hernández, J., Calvet, N., Hartmann, L., Briceño, C., Sicilia-Aguilar, A., \& Berlind, P. 2005, $A J, 129,856$

Hubeny, I. \& Lanz, T. 1995, ApJ, 439, 875

Martayan, C., Floquet, M., Hubert, A. M., Neiner, C., Frémat, Y., Baade, D., \& Fabregat, J. 2008, A\&A A 489, 459

Martayan, C., Baade, D., \& Fabregat, J. 2009, A $\mathscr{G} A$, in press (arXiv:0909.2303)

Momany, Y., et al. 2001, A\&\&A, 379, 436

Pasquini, L., et al. 2002, ESO Messenger, 110, 1

Schaller, G., Schaerer, D., Meynet, G., \& Maeder, A. 1992, A\&\&AS, 96, 269 